\title{
Production of isobutyric acid from methanol
} by Clostridium Luticellarii

\author{
Camille Petrognani ${ }^{a} b^{*}$, Nico Boon ${ }^{a, b}$, Ramon Ganiguéa ${ }^{a, b}$.
}

* presenter, Petrognani.Camille@UGent.be; ${ }^{a}$ Center for Microbiology

Ecology and Technology (CMET), Ghent University, Coupure Links 653, 9000 Ghent, Belgium; ${ }^{b}$ CAPTURE, www.capture-resources.be

\section{HIGHLIGHTS:}

- Clostridium luticellarii can produce isobutyric acid from methanol

- Supplementation of acetic and butyric acid as electron acceptors enhanced isobutyric acid titer, selectivity and productivity

- Maximum isobutyric acid production was achieved at pH 6.50

BACKGROUND: The urgency to mitigate climate change has triggered the development of strategies to reduce $\mathrm{CO}_{2}$ emissions, including microbial technologies to convert $\mathrm{CO}_{2}$ into multi-carbon products. ${ }^{1,2} \mathrm{CO}_{2}$ fermentation is hampered by low gas-liquid mass transfer due to the low solubility of $\mathrm{H}_{2}{ }^{3,4} \mathrm{CO}_{2}$-derived methanol can serve as alternative feedstock, circumventing the solubility issue..$^{4,5}$ Some acetogens can use methanol as electron donor, with a product spectrum dominated by acetic and butyric acid. ${ }^{5,6}$ The product portfolio of methanol fermentation has recently been expanded by the observation that isobutyric acid (iC4) was produced by mixed culture $\left(2.0 \mathrm{~g} \cdot \mathrm{L}^{-1} \cdot \mathrm{d}^{-1}\right) \cdot{ }^{7}$ A recent study of the ecology of a similar system revealed that the microbiome was dominated by Eubacterium and Clostridium spp., and that isobutyric acid production was closely linked to the abundance of the Clostridium sp.. ${ }^{8}$ This study isolated the organism responsible for isobutyric acid production, explored its capacity to produce isomers from a broad range of substrates and investigated potential metabolic-triggers to isomerisation, such as $\mathrm{pH}$ and electron acceptor availability.

RESULTS \& DISCUSSION: Here we obtained seven isolates that exhibited iC4 production ranging from $2.22 \mathrm{~g} . \mathrm{L}^{-1}$ to $3.90 \mathrm{~g} . \mathrm{L}^{-1}$ from an in-house isobutyric acid-producing CSTR. The 16S rRNA gene sequence analysis of the isobutyric acid-producing isolates revealed that they all shared high similarity with C. luticellarii DSM 29923.

C. Iuticellarii DSM 29923 ability to produce iC4 from different carbon sources (i.e. glucose, glycerol, methanol, ethanol, lactic acid and $\mathrm{CO} 2 \& \mathrm{H}_{2}$ ) was screened. Growth was supported on all carbon sources except ethanol, and C. Iuticellarii produced iC4 only when grown on methanol and acetic acid ( $\left.1.51 \pm 0.07 \mathrm{~g} . \mathrm{L}^{-1}\right)$ and $\mathrm{CO}_{2} \& \mathrm{H}_{2}\left(0.12 \pm 0.01 \mathrm{~g} . \mathrm{L}^{-1}\right)$. Subsequently, $C$. luticellarii ability to produce other isocarboxylic acids was explored with methanol (200 mM) as electron donor in combination with different carboxylic acids as electron acceptors. The production of other isocarboxylic acids was not detected under the tested conditions. However, C. luticellarii 
was shown to be able to synthesise valeric and caproic acid with acetic and propionic as electron acceptor, respectively. Here, we also screened the effect of $\mathrm{pH}$ on iC4 production by $\mathrm{C}$. luticellarii. Over the range of $\mathrm{pH} 5.50$ to 6.50 , increasing $\mathrm{pH}$ led to higher cell densities and iC4 production (from $0.37 \pm 0.02 \mathrm{~g} . \mathrm{L}^{-1}$ to $\left.3.91 \pm 0.06 \mathrm{~g} . \mathrm{L}^{-1}\right)$. Further increasing the $\mathrm{pH}$ up to 7.00 resulted in higher iC4 selectivity ( $83 \%$ at $\mathrm{pH} 7.00)$, but the methanol converted was halved.

Finally, the evolution of the product spectrum was monitored throughout the batch incubation under three selected conditions (M, MA and MAB, Figure 1). During growth on methanol with $\mathrm{CO}_{2}$ as only available electron acceptor (M), acetic and butyric acid production started immediately. iC4 production only started after six days when $C$. luticellarii started consuming the acetic acid produced in-situ. When acetic acid was fed as an electron acceptor in condition MA, butyric and iC4 were directly coproduced. Under the condition with butyric acid supplementation (MAB), only iC4 net production occurred during the first six days. Following day six, butyric acid started to accumulate. Overall, it was observed that the supplementation of acetic and butyric acid also enhanced iC4 production in terms of production rate (from $0.120 \pm 0.024 \mathrm{~g} . \mathrm{L}^{-1} . \mathrm{d}^{-1}$ in condition M to $0.420 \pm 0.012 \mathrm{~g} \cdot \mathrm{L}^{-1} . \mathrm{d}^{-}$ 1 in condition MAB). Additionally, condition MA revealed that preliminary accumulation of butyric acid is not necessary to trigger isobutyric acid production.
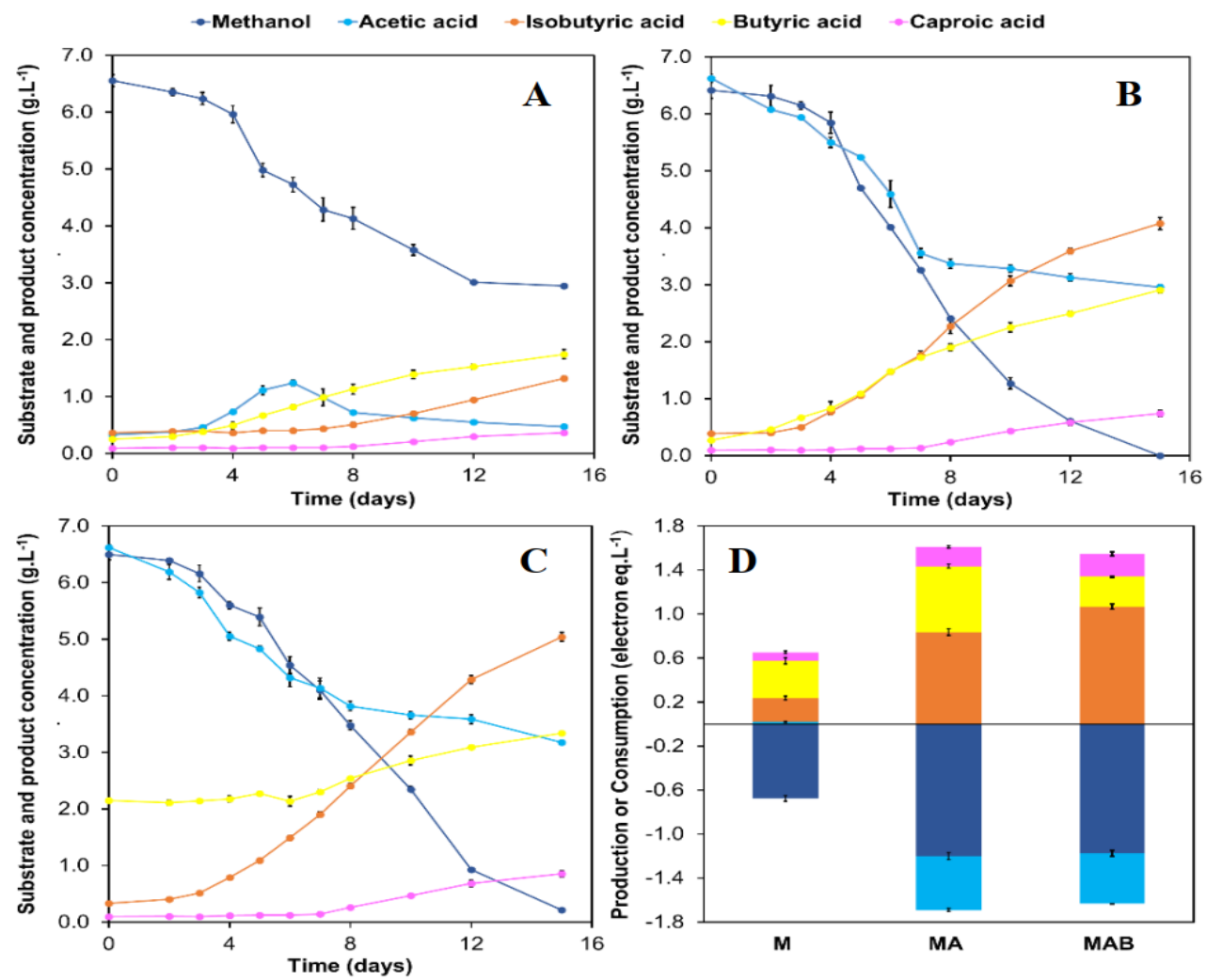

Figure 1. Substrate and product concentration profile during batch growth of C. luticellarii DSM 29923 on methanol (Panel A; M), and methanol and acetic acid with (Panel $C$; MAB) and without (Panel B; MA) butyric acid supplementation. Panel $D$ represents the electron balance. 
CONCLUSION: Clostridium luticellarii can produce isobutyric acid from C1 carbon sources, including methanol. It can generate isobutyric acid without the external addition of acetic and butyric acid as electron acceptors, although their presence steered isobutyric acid production in terms of final titer, selectivity, and productivity (maximum of $5.04 \pm 0.08 \mathrm{~g} . \mathrm{L}-1,70 \%$ and $0.420 \pm 0.012$ g.L-1.d-1, respectively). $\mathrm{pH}$ was shown to significantly influence isobutyric acid production with the highest production obtained at $\mathrm{pH} 6.50$.

\section{REFERENCES}

1. SAPEA, Novel carbon capture and utilisation technologies: research and climate aspect, 2018, DOI:10.26356/CARBONCAPTURE.

2. Q. Zhu, Developments on CO2-utilization technologies, Clean Energy, 2019, 3, 85-100.

3. A. Satanowski and A. Bar-Even, A one-carbon path for fixing $\mathrm{CO} 2$, EMBO Rep., 2020, DOI:10.15252/embr.202050273.

4. A. Prévoteau, J. M. Carvajal-Arroyo, R. Ganigué and K. Rabaey, Microbial electrosynthesis from CO2: forever a promise?, Curr. Opin. Biotechnol., 2020, 62, 48-57.

5. E. A. Quadrelli, G. Centi, J.-L. Duplan and S. Perathoner, Carbon Dioxide Recycling: Emerging Large-Scale Technologies with Industrial Potential, ChemSusChem, 2011, 4, 1194-1215.

6. R. Takors, M. Kopf, J. Mampel, W. Bluemke, B. Blombach, B. Eikmanns, F. R. Bengelsdorf, D. Weuster-Botz and P. Dürre, Using gas mixtures of $\mathrm{CO}, \mathrm{CO} 2$ and $\mathrm{H} 2$ as microbial substrates: the do's and don'ts of successful technology transfer from laboratory to production scale, Microb. Biotechnol., 2018, 11, 606-625.

7. W.-S. Chen, S. Huang, D. P. Strik and C. J. Buisman, Isobutyrate biosynthesis via methanol chain elongation: converting organic wastes to platform chemicals, J. Chem. Technol. Biotechnol., 2017, 92, 13701379.

8. S. Huang, R. Kleerebezem, K. Rabaey and R. Ganigué, Open microbiome dominated by Clostridium and Eubacterium converts methanol into i-butyrate and n-butyrate, Appl. Microbiol. Biotechnol., 2020, DOI: $10.1007 /$ s00253-020-10551-w. 